\title{
Thermal Oxidation of Structured Silicon Dioxide
}

Christiansen, Thomas Lehrmann; Hansen, Ole; Jensen, Jørgen Arendt; Thomsen, Erik Vilain

Published in:

E C S Journal of Solid State Science and Technology

Link to article, DOI:

$10.1149 / 2.003405 j$ ss

Publication date:

2014

Document Version

Publisher's PDF, also known as Version of record

Link back to DTU Orbit

Citation (APA):

Christiansen, T. L., Hansen, O., Jensen, J. A., \& Thomsen, E. V. (2014). Thermal Oxidation of Structured Silicon Dioxide. E C S Journal of Solid State Science and Technology, 3(5), N63-N68.

https://doi.org/10.1149/2.003405jss

\section{General rights}

Copyright and moral rights for the publications made accessible in the public portal are retained by the authors and/or other copyright owners and it is a condition of accessing publications that users recognise and abide by the legal requirements associated with these rights.

- Users may download and print one copy of any publication from the public portal for the purpose of private study or research.

- You may not further distribute the material or use it for any profit-making activity or commercial gain

- You may freely distribute the URL identifying the publication in the public portal 


\title{
Thermal Oxidation of Structured Silicon Dioxide
}

\author{
Thomas Lehrmann Christiansen, ${ }^{\text {a,z }}$ Ole Hansen, ${ }^{\text {a,b }}$ Jørgen Arendt Jensen, ${ }^{c}$ \\ and Erik Vilain Thomsen ${ }^{\mathrm{a}}$
}

\author{
${ }^{a}$ Department of Micro- and Nanotechnology, Technical University of Denmark, DK-2800 Kgs. Lyngby, Denmark \\ ${ }^{b}$ Center for Individual Nanoparticle Functionality CINF, Technical University of Denmark, \\ DK-2800 Kgs. Lyngby, Denmark \\ ${ }^{c}$ Center for Fast Ultrasound Imaging, Department of Electrical Engineering, Technical University of Denmark, \\ DK-2800 Kgs. Lyngby, Denmark
}

\begin{abstract}
The topography of thermally oxidized, structured silicon dioxide is investigated through simulations, atomic force microscopy, and a proposed analytical model. A $357 \mathrm{~nm}$ thick oxide is structured by removing regions of the oxide in a masked etch with either reactive ion etching or hydrofluoric acid. Subsequent thermal oxidation is performed in both dry and wet ambients in the temperature range $950^{\circ} \mathrm{C}$ to $1100^{\circ} \mathrm{C}$ growing a $205 \pm 12 \mathrm{~nm}$ thick oxide in the etched mask windows. Lifting of the original oxide near the edge of the mask in the range $6 \mathrm{~nm}$ to $37 \mathrm{~nm}$ is seen with increased lifting for increasing processing temperatures. Oxides structured by reactive ion etching are lifted on average a factor of four more than oxides etched in hydrofluoric acid. Both simulations and the analytical model successfully predict the oxide topography qualitatively, showing that the mask lifting phenomenon is governed mainly by diffusion and the geometry of the oxide. Simulations also predict the oxide topography quantitatively, with an average root mean square deviation of $1.2 \mathrm{~nm}$ and a maximum deviation of $13 \mathrm{~nm}(39 \%)$ from the mean of the measured values.

(c) 2014 The Electrochemical Society. [DOI: 10.1149/2.003405jss] All rights reserved.
\end{abstract}

Manuscript submitted December 13, 2013; revised manuscript received February 20, 2014. Published March 5, 2014.

Thermal oxidation of silicon is a well-known and widely used process in the microfabrication of semiconductor devices. Silicon dioxide exhibits excellent electrical and mechanical properties and has a highly predictable growth rate, which has made thermal oxidation an indispensable process in the semiconductor industry.

The growth of silicon dioxide on planar silicon surfaces is well described by the Deal and Grove model, which accurately predicts the growth of silicon dioxides with the exception of very thin oxides $(<250 \AA)$ grown in a dry ambient. ${ }^{1}$ The latter case was treated by Massoud et al., who introduced an exponentially decaying addition to the growth rate, thereby correcting for the faster initial oxidation rate of thin oxides. ${ }^{2}$

However, for non-planar silicon surfaces, or silicon surfaces with structured thin films, e.g. thin films subjected to a masked etch, the oxidation can no longer be described as a one-dimensional problem in the vicinity of the surface discontinuity. Such situations are frequently encountered, e.g. in the oxidation of silicon surfaces structured by etching and in LOCOS oxidations, where nitride diffusion barriers are exploited to produce selective oxidation of pre-defined regions of the silicon surface. The complex shape of the oxide grown in the vicinity of such nitride masks, known as the bird's beak, has been investigated intensively in the literature due to its widespread use in the fabrication of thick insulation oxides. ${ }^{3,4,5}$ Oxidation of structured silicon has also attracted some attention, primarily due to the unwanted artifacts that arise near corners, such as oxide thinning,,${ }^{6,7,8}$ rounding or sharpening of convex corners, ${ }^{9}$ and oxide protrusions arising from oxidation of $90^{\circ}$ silicon corners. ${ }^{10}$

Thermal oxidation of structured silicon dioxide has so far only been studied sparingly in literature. ${ }^{11}$ This paper investigates the oxidation of an oxide step, a situation which arises if e.g. a thermally grown oxide is structured by etching and subsequently oxidized a second time in order to grow an oxide in the etched mask windows. The structured oxide acts as a diffusion mask during the second oxidation, and will experience lifting in the vicinity of the mask edge due to 2-D oxidation effects as shown in Fig. $1 .{ }^{12,13}$ Such mask lifting can pose a problem if the flatness of the oxide surface is critical, e.g. if direct bonding is involved in subsequent processing steps. ${ }^{14,15}$

In this paper, the topography of the oxide mask is investigated as a function of processing parameters through simulations, measurements, and analytical considerations. The study considers a fixed initial oxide mask thickness of $357 \mathrm{~nm}$, in which mask windows are opened using either reactive ion etching or hydrofluoric acid. For both types of mask windows, a second oxidation is performed, aiming for a

${ }^{\text {zE} E-m a i l: ~ t l e h r @ n a n o t e c h . d t u . d k ~}$
$200 \mathrm{~nm}$ oxide thickness in the un-masked part of the wafer. By fixing the thickness of the two oxidations, the effect of processing parameters is investigated by carrying out the second oxidation at different oxidation temperatures from $950^{\circ} \mathrm{C}$ to $1100^{\circ} \mathrm{C}$ for both wet and dry oxidations.

Good agreement is seen between simulations and measurements, demonstrating increased mask lifting at increasing oxidation temperatures and significantly larger mask lifting for mask windows etched by reactive ion etching compared to those etched using hydrofluoric acid. This trend is confirmed by a proposed analytical model, which demonstrates that the mask lifting is essentially a function of the thicknesses of the oxides far from the oxide step. Stress effects, which are known to have a large influence on the geometry of the bird's beak, ${ }^{5}$ are seen to be negligible for the oxide step in the temperature ranges considered in this study.

The structure of the paper is as follows: First, the theory describing the thermal oxidation of an oxide step geometry is covered. The mathematical details of the conformal mapping technique used to solve the problem analytically is presented in the appendix for convenience. This is followed by a description of the experimental setup, including sample preparation and measurement procedure. Subsequently, the approach used in the simulations to emulate the experiments is covered prior to a presentation and discussion of the results.

\section{Theory}

The oxide growth in the vicinity of an oxide step can be modeled under the assumption that the oxidation rate is diffusion limited. In the

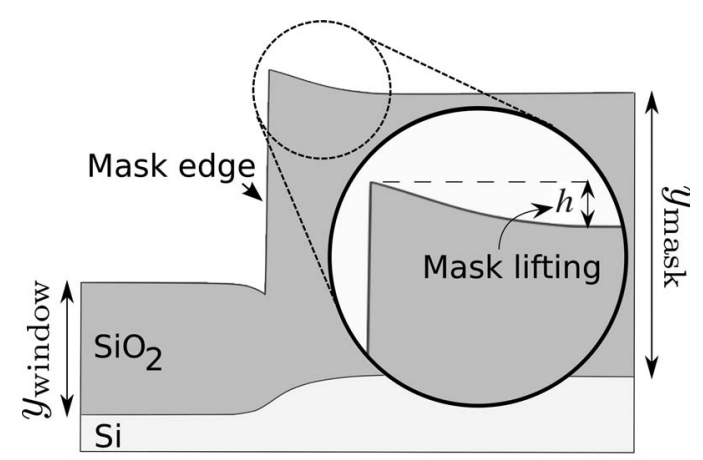

Figure 1. Simulated oxide geometry after thermal oxidation of an oxide step showing the mask lifting near the mask edge. 


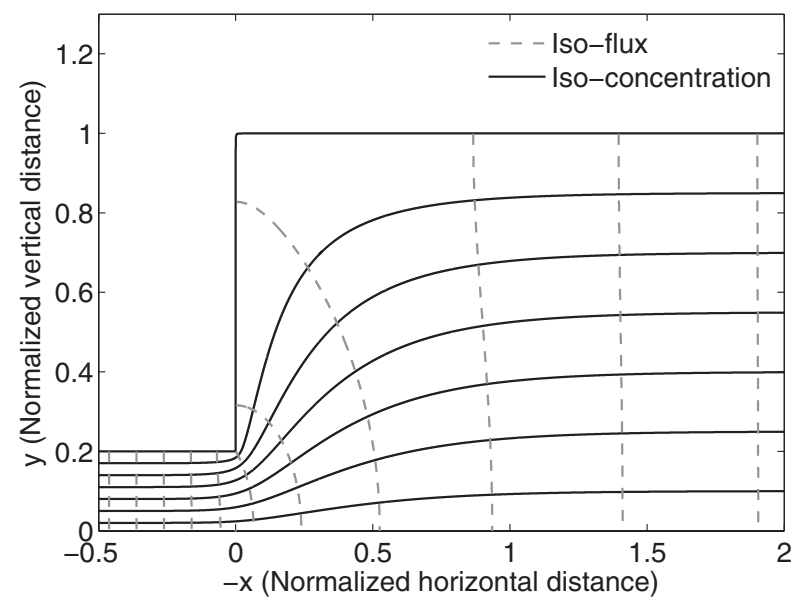

Figure 2. Iso-concentration and iso-flux lines in an oxide step geometry calculated using the conformal mapping approach. The distances are normalized to the thickness of the mask oxide (to the right of the step).

oxide film, the continuity equation governs the transport of the oxidant. For realistic diffusion times $t>y_{\mathrm{ox}}^{2} /(4 D)$, where $y_{\mathrm{ox}}$ is the thickness of the oxide and $D$ is the diffusivity, the diffusion will be almost stationary, such that the time derivative of the oxidant concentration $C$ becomes

$$
\frac{\partial C}{\partial t}=D \nabla^{2} C \simeq 0
$$

where $\nabla^{2}$ denotes the Laplace operator. Hence, the oxide growth rate can be expressed directly as a function of the flux density normal to the silicon/oxide interface, $J$,

$$
\frac{d s}{d t}=\frac{J}{N}
$$

where $s$ is the oxide thickness at time $t$ at the silicon/oxide interface, and $N$ is the number of oxidizer molecules incorporated per unit volume of the oxide. Thus, if the normal incident flux density at the interface can be found, the oxide thickness can be calculated by a time integration of Eq. (2).

This can be achieved by use of conformal mapping, covered in detail in the appendix, with which the flux- and concentration distribution in the structured oxide can be calculated. As an example, Fig. 2 shows the iso-concentration and iso-flux contour lines of an oxide step with a thickness ratio of $y_{\text {mask }} / y_{\text {window }}=5$, where $y_{\text {window }}$ is the thickness of the oxide grown in the mask windows and $y_{\text {mask }}$ is the thickness of the oxide mask. The definition of these thicknesses are given in Fig. 1. The flux density at the step, $J_{0}$, is in the derivation in the appendix shown to be proportional to the flux density in the mask windows, $J_{\text {window }}$,

$$
J_{0} \simeq 0.83 J_{\text {window }} .
$$

The ratio of the oxide grown at the step to the oxide thickness grown in the mask window far from the step, $\beta$, thus becomes a constant, since

$$
\beta=\frac{\int_{0}^{t_{0}} J_{0}(t) / N d t}{\int_{0}^{t_{0}} J_{\text {window }}(t) / N d t}=0.83 \frac{\int_{0}^{t_{0}} J_{\text {window }}(t) / N d t}{\int_{0}^{t_{0}} J_{\text {window }}(t) / N d t}=0.83 .
$$

The lifting of the mask at the mask edge at time $t$, denoted $h$ in Fig. 1, may then simply be expressed as

$$
h(t)=0.56\left\{\beta y_{\text {window }}(t)-\left[y_{\text {mask }}(t)-y_{\text {mask }}(0)\right]\right\},
$$

where the factor of 0.56 accounts for the volume expansion by a factor of 2.2 of the growing oxide compared to the consumed silicon. ${ }^{17}$

It is seen from Eq. (5) that the mask lifting becomes a function only of the oxide thicknesses far from the step, which may be found
Table I. Parameters for the oxidation of $\operatorname{Si}(100) .{ }^{18,19}$

\begin{tabular}{lcccc} 
& $\begin{array}{c}B_{0} \\
{\left[\mu \mathrm{m}^{2} \mathrm{~min}^{-1}\right]}\end{array}$ & $\begin{array}{c}E_{a, B} \\
{[\mathrm{eV}]}\end{array}$ & $\begin{array}{c}\frac{B_{0}}{A_{0}} \\
{\left[\mu \mathrm{m} \mathrm{min}^{-1}\right]}\end{array}$ & $\begin{array}{c}E_{a, B / A} \\
{[\mathrm{eV}]}\end{array}$ \\
\hline Dry oxidation & 12.8667 & 1.23 & $6.176 \times 10^{4}$ & 2.0 \\
Wet oxidation & 7.00 & 0.78 & $1.755 \times 10^{5}$ & 2.05
\end{tabular}

using Deal and Grove's model. ${ }^{1}$ This states that the thickness of the growing oxide at time $t$ is

$$
y_{\mathrm{ox}}=\sqrt{\frac{A^{2}}{4}+B(t+\tau)}-\frac{A}{2},
$$

where $\tau$ is the time shift induced by an already existing oxide. The constants $A$ and $B$ are found via the parabolic and linear rate constants, $B$ and $B / A$, given by

$$
\begin{gathered}
B=B_{0} \exp \left(-\frac{E_{a, B}}{k_{B} T}\right), \\
\frac{B}{A}=\frac{B_{0}}{A_{0}} \exp \left(-\frac{E_{a, B / A}}{k_{B} T}\right),
\end{gathered}
$$

where $k_{B}$ is Boltzmann's constant, $T$ is the absolute temperature, $E_{a, B}$ and $E_{a, B / A}$ are activation energies, and $B_{0}$ and $B_{0} / A_{0}$ are the parabolic and linear rate constants in the infinite temperature limit. The values relevant for this study are given in Table $\mathrm{I} .{ }^{18,19}$ The partial pressure, $P$, of the oxidant during the oxidation influences the parabolic and linear rate constants, such that for a reference pressure of $P_{0}=1 \mathrm{~atm}$, the pressure dependence is given by 19,20

$$
\begin{gathered}
\frac{B}{A}(P)=\frac{B}{A} \frac{P}{P_{0}}, \\
B(P)=B \frac{P}{P_{0}} .
\end{gathered}
$$

From Eq. (5), the mask lifting's dependency on processing parameters can be deduced. In this study, the initial mask oxide thickness, $y_{\text {mask }}(0)$, is kept constant, and the final thickness of the oxide grown in the mask window, $y_{\text {window }}$, is targeted at $200 \mathrm{~nm}$, such that this can be assumed a constant as well. Thus, the only variable is the final mask oxide thickness, with thicker mask oxides yielding smaller mask lifting. The thickness of the oxide can be described by Eq. (6). In the limit of large values of $t+\tau$, where the oxidation time $t$ is dictated by the desired thickness of the oxide in the mask windows, and $\tau$ is dictated by the initial mask oxide thickness, Eq. (6) reduces to

$$
y_{\mathrm{ox}} \simeq \sqrt{B(t+\tau)}, \quad t+\tau \gg 0 .
$$

The final oxide mask thickness therefore becomes

$$
y_{\text {mask }}=\sqrt{y_{\text {window }}\left[y_{\text {window }}+A\right]+y_{\text {mask }}(0)\left[y_{\text {mask }}(0)+A\right]} \text {. }
$$

If both $y_{\text {window }}$ and $y_{\text {mask }}(0)$ are constants, the mask lifting depends only on the constant $A$, which relates to the oxidant diffusivity $D$ and surface reaction rate $k$ as $A=2 D / k .^{1}$ In Fig. 3, $A$ has been plotted for both dry and wet oxidations in the temperature range relevant for this study. It is seen that $A$ decreases for increasing temperature, and thus the final mask oxide thickness will decrease for increasing temperature. From Eq. (5), it is seen that this results in an increased mask lifting. This is true for both wet and dry oxidations. However, $A$ decreases more rapidly for increasing temperatures for wet oxidations, indicating that the mask lifting will depend more strongly on temperature for wet oxidations compared to dry oxidations. In addition to this, $A$ will, for a given temperature, be larger for wet oxidations than for dry oxidations, yielding a larger mask lifting for dry oxidations.

\section{Experimental}

Sample preparation. - All samples in this study were made using a total of six $525 \pm 25 \mu \mathrm{m}$ single side polished boron doped (100) $4^{\prime \prime}$ 


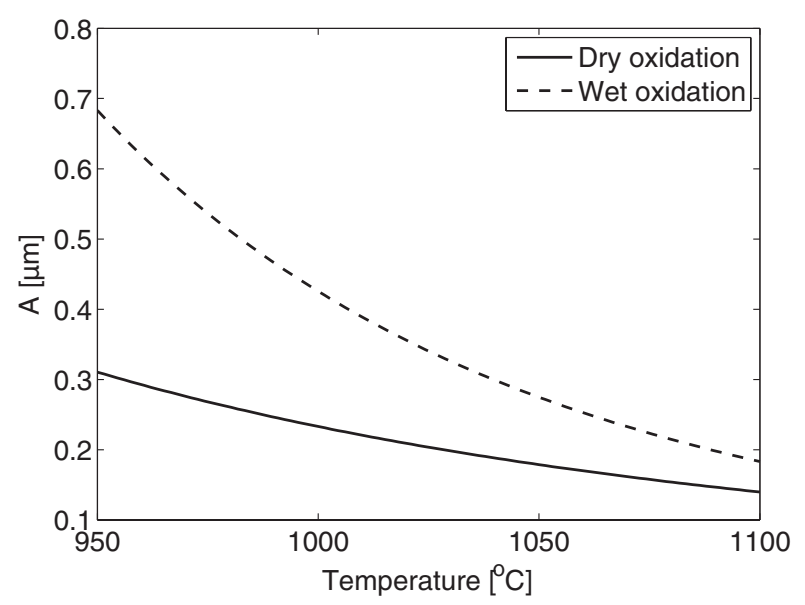

Figure 3. Plot of the constant $A$ as a function of temperature for both dry and wet oxidations.

silicon wafers with a resistivity of $1-10 \Omega-\mathrm{cm}$. To achieve a uniform oxide mask thickness on all samples, the six wafers were oxidized simultaneously using a Tempress horizontal furnace in a dry oxygen ambient at $1100^{\circ} \mathrm{C}, 1 \mathrm{~atm}$, for 6 hours and 40 minutes, yielding an oxide mask thickness of $357 \pm 1 \mathrm{~nm}$. The thickness was measured by fitting the reflectance spectrum at zero degrees and 70 degrees angle of incidence. All wafers were then treated with hexamethyldisilazane (HMDS) prior to a lithography step on the polished side of the wafers using a $2.2 \mu \mathrm{m}$ thick AZ 5214E photoresist and an AZ 351B developer. The photoresist was exposed to UV light for 9 seconds through a chromium/soda-lime glass mask with arrays of squares having a side length of $65 \mu \mathrm{m}$. In these squares, the oxide mask windows were opened using an anisotropic reactive ion etch (RIE) for the first three wafers and a buffered hydrofluoric acid (BHF) wet etch for the remaining three. This was done to enable investigation of the influence of the shape of the mask edge. The photoresist was subsequently stripped in an $\mathrm{O}_{2} / \mathrm{N}_{2}$ RF plasma with gas flows of $240 / 70 \mathrm{sccm}$ operating at $1000 \mathrm{~W}$ for 25 minutes.

Each of the six wafers were then cleaved in half with a diamond scriber, yielding a total of 12 samples that were cleaned using a 10 minute clean in a $\mathrm{NH}_{4} \mathrm{OH}: \mathrm{H}_{2} \mathrm{O}_{2}: \mathrm{H}_{2} \mathrm{O}(1: 1: 5)$ mixture followed by a 10 minute clean in a $\mathrm{HCl}: \mathrm{H}_{2} \mathrm{O}_{2}: \mathrm{H}_{2} \mathrm{O}(1: 1: 5)$ mixture, both heated to $70^{\circ} \mathrm{C}$. All samples were subsequently oxidized in a Tempress horizontal furnace using the processing temperatures listed in Table II. Each sample has been assigned a number from 1 to 12 , which will be used as identification in the remainder of this paper. All oxidations included a 20 minute post-annealing step in an inert nitrogen atmosphere at the given oxidation temperature. All dry oxidations were performed at $1 \mathrm{~atm}$, while the wet oxidations were performed at $0.8 \mathrm{~atm} \mathrm{H}_{2} \mathrm{O}$ partial pressure as dictated by the settings of the furnace. The oxidation times for the respective oxidation temperatures were chosen such that a final thickness of roughly $200 \mathrm{~nm}$ was achieved in the mask windows. Silicon dummy wafers were included in each oxidation, allowing the actual oxide thickness to be measured, the values of which are listed in Table III. Note that a total of six oxidations

Table II. Table of the processing parameters used for the twelve samples, numbered from 1 to 12 .

Oxidation temp. $\left[{ }^{\circ} \mathrm{C}\right]$

\begin{tabular}{lccccc}
\cline { 3 - 6 } Mask etch & Ox. type & 950 & 1000 & 1050 & 1100 \\
\hline RIE & Dry & 1 & 2 & 3 & 4 \\
RIE & Wet & 5 & 6 & - & - \\
BHF & Dry & 7 & 8 & 9 & 10 \\
BHF & Wet & 11 & 12 & - & -
\end{tabular}

Table III. Thicknesses of the oxides grown during the second oxidation.

Oxidation temperature $\left[{ }^{\circ} \mathrm{C}\right]$

\begin{tabular}{lcccc}
\cline { 2 - 5 } Ox. type & 950 & 1000 & 1050 & 1100 \\
\hline Dry & $195 \mathrm{~nm}$ & $210 \mathrm{~nm}$ & $217 \mathrm{~nm}$ & $217 \mathrm{~nm}$ \\
Wet & $192 \mathrm{~nm}$ & $204 \mathrm{~nm}$ & - & -
\end{tabular}

were carried out, each containing two samples; one with a RIE etched mask and one with a BHF etched mask. No wet oxidations were performed at $1050^{\circ} \mathrm{C}$ and $1100^{\circ} \mathrm{C}$, as the required oxidation time to reach $200 \mathrm{~nm}$ is impractically short ( $<15$ minutes), potentially resulting in a less well-defined and homogeneous oxide.

Measurements. - The oxide mask topography was measured using atomic force microscopy (AFM) using an approach similar to the one used by Sarioglu et al. ${ }^{10}$ AFM was chosen over other possible measurement equipment such as scanning electron microscopy and transmission electron microscopy due to the aspect ratio of the investigated features, containing height differences less than $50 \mathrm{~nm}$ evolving over a distance in the order of microns. All AFM measurements were made using a Nanoman atomic force microscope operating in tapping mode, scanning a $5 \mu \mathrm{m}$ by $5 \mu \mathrm{m}$ area in 512 parallel scan lines covering the mask edge. An example of such a scan is shown in Fig. 4, corresponding to sample no. 3. The scan direction indicated in Fig. 4, was incorporated to avoid measurement artifacts such as overshooting from the abrupt step at the mask edge. Three such scans were performed on each sample to take processing variations into account, resulting in a total of 1536 scan lines per sample. Each scan line was post-processed in MATLAB (MathWorks, Inc., Natick, MA, USA) subject to the following routine:

1 The scan line was leveled by subtracting a linear function with a slope determined from two points on the oxide mask: $1 \mu \mathrm{m}$ from the mask edge and the point farthest away from the mask edge.

2 The height of the point on the mask farthest away from the mask edge was subtracted from all points on the scan line to set this value to zero.

3 The scan lines were shifted horizontally such that the points with maximum height (corresponding to the mask edge) were aligned.

This routine was carried out to account for drift in the AFM measurements, thereby allowing for comparison of the individual profiles. Each profile measurement reported in this study is the average value

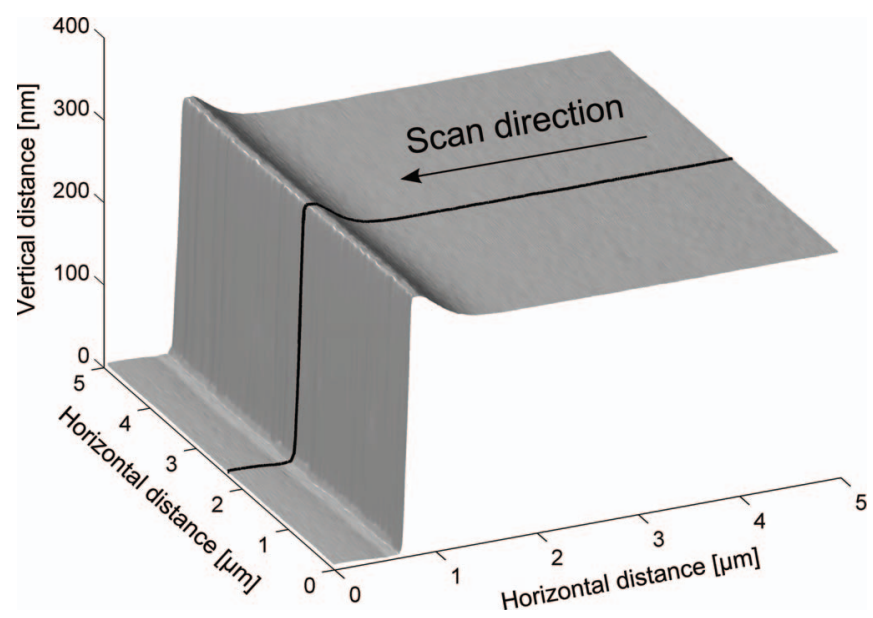

Figure 4. Illustration showing an AFM scan of sample no. 3 with 512 scan lines. A single scan line has been marked in black with an indication of the scan direction incorporated to avoid overshooting artifacts at the oxide mask edge. 


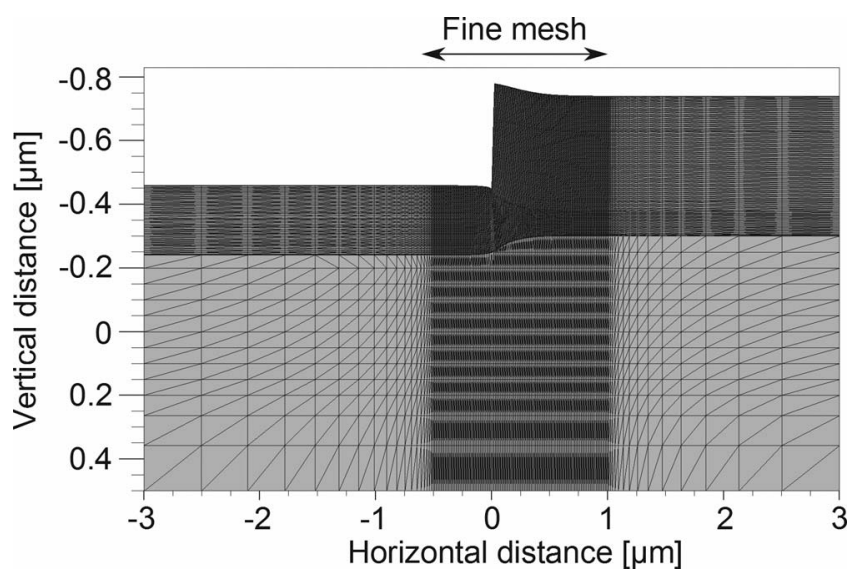

Figure 5. Simulation of the oxidation of sample no. 3 showing the mesh used for all simulations. The mesh is finer in the horizontal direction next to the oxide step where 2-D oxidation effects are prominent.

of these 1536 postprocessed scans. The standard deviation of the $j$ 'th measurement point of a profile representing a given sample is found as

$$
\sigma(j)=\sqrt{\frac{1}{N-1} \sum_{i=1}^{N}\left[x_{i}(j)-\bar{x}(j)\right]^{2}},
$$

where $x_{i}(j)$ is the average of the $j$ 'th measurement point taken over the 512 scan lines of the $N=3$ measurement sites on each sample, and $\bar{x}(j)$ is the global average of the $j$ 'th measurement point taken over all 1536 scan lines from the sample. This standard deviation thus reflects processing variations over the whole sample. The standard deviation within a single measurement containing 512 scan lines was in all cases below $1 \mathrm{~nm}$, corresponding to the roughness of the sample. This small variation is negligible compared to the variation between the three measurement sites and has therefore not been included in the presentation of the data.
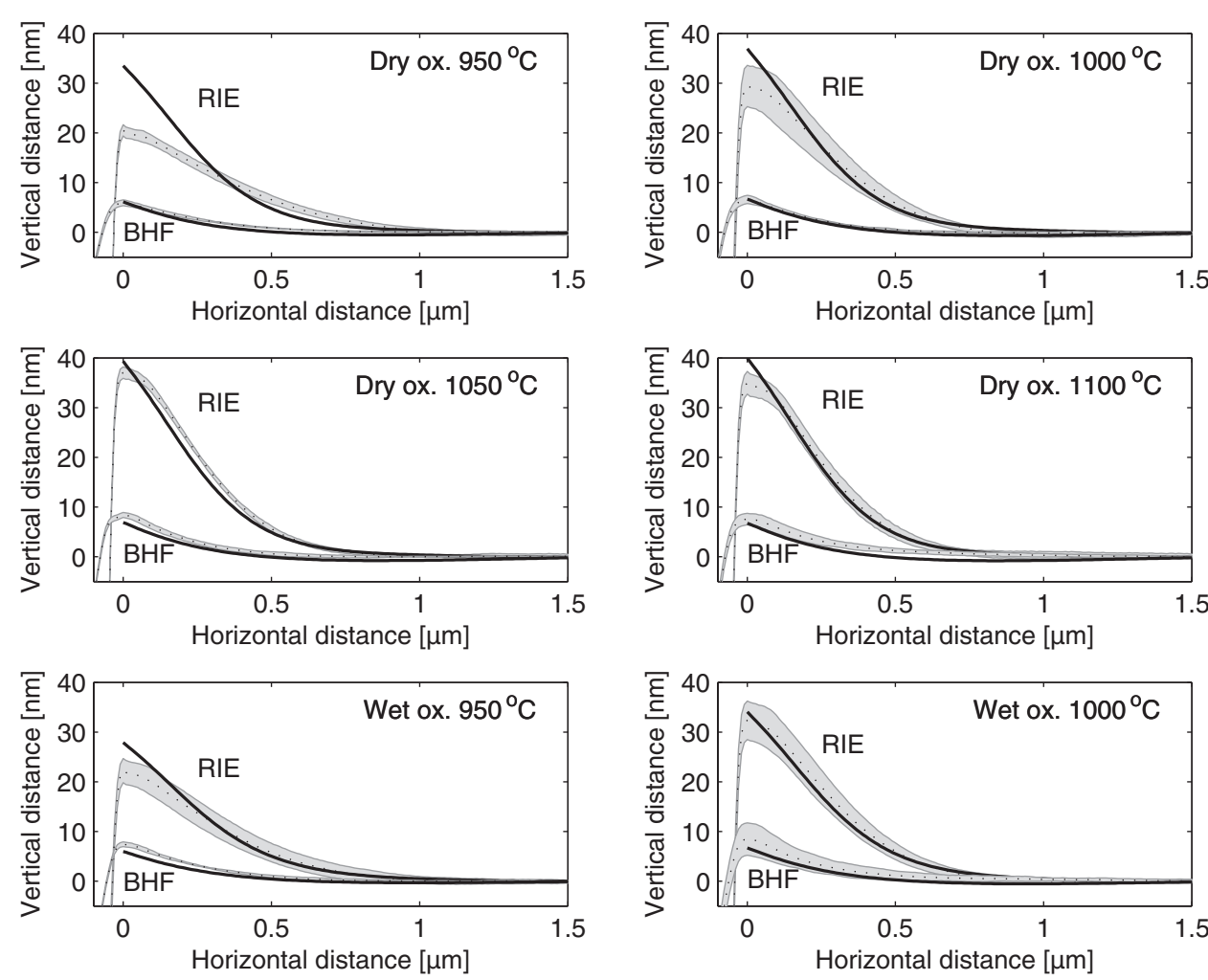

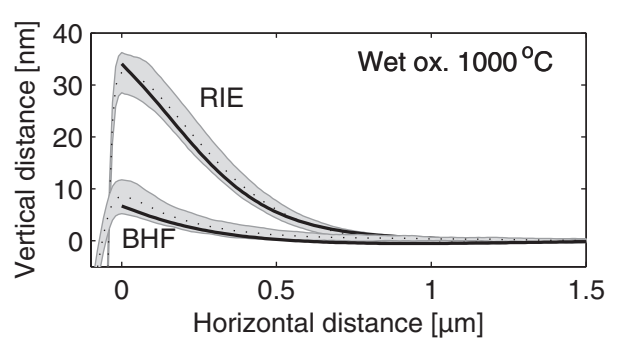

\section{Simulations}

The simulations were carried out in Athena 5.20.0.R (Silvaco Inc., Santa Clara, CA, USA) using the same oxidation parameters in terms of type, final oxide thickness, temperature, pressure, and annealing as were used in the experiments. The initial structure for the simulations included a region $3 \mu \mathrm{m}$ to each side of the oxide mask as illustrated in Fig. 5, showing the case of a RIE etched mask after the second oxidation. In the horizontal direction, a mesh size of $10 \mathrm{~nm}$ was used in the interval $[-0.5 \mu \mathrm{m}, 1 \mu \mathrm{m}]$, the oxide mask edge being located at $x=0$. Outside this region, where 2 -D oxidation effects are negligible, the mesh size was set to increase linearly to $500 \mathrm{~nm}$ at the boundary of the simulation.

The resolution of the mesh created during the oxidation was set to $5 \mathrm{~nm}$. This resolution was found by simulating a dry oxidation at $950^{\circ} \mathrm{C}$ for 880 minutes using a mesh size of $100 \mathrm{~nm}, 50 \mathrm{~nm}$, $10 \mathrm{~nm}, 5 \mathrm{~nm}$, and $1 \mathrm{~nm}$, respectively. The relative change in the final oxide thickness stagnated to a value below $0.5 \%$ when changing from $5 \mathrm{~nm}$ to $1 \mathrm{~nm}$, hence $5 \mathrm{~nm}$ was chosen for giving both sufficient accuracy and computational speed. The simulations were carried out using the "Viscous" model, which includes both viscous flow of the oxide and stress. However, the simulations were also carried out using the "Compress" model that excludes stress, yielding identical results, thereby indicating negligible stress effects. ${ }^{21}$ This fact is supported by the work of EerNisse, who demonstrated that thermal oxides grow in stress-free conditions at temperatures above $950-975^{\circ} \mathrm{C}$, while large compressive stresses arise during growth for temperatures lower than this. $^{22}$

The RIE etched masks were simulated by a perfect right angle mask etch, while the BHF etched profile was extracted from the actual measured profile. The latter method was not used for the RIE etched masks, as the step was too abrupt to be resolved by the AFM.

\section{Results and Discussion}

Fig. 6 shows the measured and simulated profiles of the oxide mask in the vicinity of the mask edge after the second oxidation. Each plot shows the result for a given temperature at either dry or wet oxidation.
Figure 6. Simulated and measured profiles of the mask surface in the vicinity of the mask edge. The dotted lines indicate the mean of the measurements while the shaded area shows \pm 2 standard deviations. The solid black lines show the simulated profiles. 
Furthermore, the results from both RIE and BHF etched oxide masks are given in each plot.

For the RIE etched oxide masks, the average root mean square (RMS) deviation of the simulated values from the mean of the measured values is $1.6 \mathrm{~nm}$. The simulated values only deviate significantly from the measured in the region just around the mask edge, where the maximum deviation from the mean for the samples 2 through 6 is $7 \mathrm{~nm}(20 \%)$. This discrepancy in the vicinity of the mask edge could be explained by either reflow of the oxide or slight differences between the perfect right angle mask edge assumed in the simulation and the actual profile created by the RIE etch. However, sample 1 is an outlier, since it deviates significantly from the measured values in a much larger region than the remaining samples, up to as much as $13 \mathrm{~nm}(39 \%)$ from the mean. This large deviation cannot be explained by the same factors, since the same RIE etch was used for all the samples 1 through 6 , and oxide reflow is expected to be more pronounced for higher temperatures. However, sample 1 was oxidized at $950^{\circ} \mathrm{C}$, which is the lower limit of stress-free oxide growth. ${ }^{22}$ The pronounced discrepancy could therefore be due to unrelieved stresses in the oxide, which the simulation fails to capture. The discrepancy was still present if stress effects were included in the simulation, but the stress behavior in this limiting case might not be well described by the simulation software.

The results for the samples 7 through 12 , corresponding to the samples with BHF etched oxide masks, are in general closer to the measured values than is seen for the samples 1 through 6 , with an average RMS deviation between the simulated values and the mean of the measured of $0.8 \mathrm{~nm}$. This is consistent with the fact that the simulation was carried out using the measured profile of the mask after the BHF etch, and thus the simulations are less vulnerable to discrepancies between the simulated and the actual mask profile. The maximum deviation between the simulated profiles and the mean of measured profiles is therefore less than $1.8 \mathrm{~nm}(26 \%)$.

In Fig. 7, the simulated and measured mask lifting at the mask edge has been extracted for each of the six oxidation temperatures and -types. The figure has been divided into four sections, equivalent to the four combinations of wet and dry oxidations, RIE and BHF mask etchings. Furthermore, for the RIE etched masks, the analytical prediction given by Eq. (5) has been plotted along with the measured and simulated values.

It is seen that the analytical prediction in general overestimates both the measured and the simulated mask lifting. The deviation from the measured values is expected, since the simulated values also overestimate these. The reason for the deviation between the analytical and the simulated values can be explained by the fact that the conformal

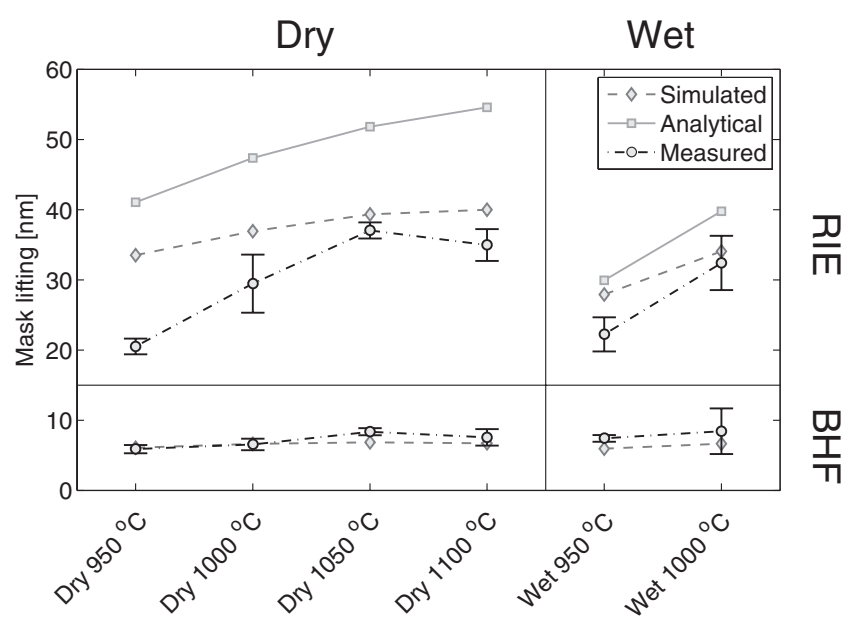

Figure 7. Mask lifting at the mask edge as a function of oxidation temperature and type for oxide mask etched by either RIE or BHF. Both measured, simulated and analytically estimated values are given. mapping, on which the theory is based, assumes a right angle oxide step and a flat oxide-silicon interface at all times, which is of course a simplifying assumption. Furthermore, the simulated profile considers the top surface of the oxide mask, while the conformal mapping only considers the oxide grown at the silicon/oxide interface, i.e. below the mask. Thus, the geometrical deformation of the mask created during growth is not captured by the theory.

Despite the mentioned discrepancies, both the simulated and the analytically estimated values of the mask lifting successfully capture the qualitative parameter dependency. As was predicted by Eq. (5), and confirmed by both simulations and measurements, the mask lifting increases with oxidation temperature. Note, however, that the temperature dependence seen in Fig. 7 is slightly misleading, since the final oxide thickness outside the mask is not exactly the same for all oxidations. Rather, it increases slightly with temperature, as seen in Table III, leading to an exaggerated temperature dependence.

The analytical estimate and the simulated values both predict that, for equal temperatures, the wet oxidations should yield smaller mask liftings than dry oxidations. Furthermore, they both predict a more pronounced temperature dependency for wet oxidations compared to dry oxidations. However, the measurements do not show significantly different mask liftings for wet and dry oxidations at $950^{\circ} \mathrm{C}$ and $1000^{\circ} \mathrm{C}$, respectively.

In general, the oxidation temperature and oxidation type are seen to have only a minor influence on the mask lifting. As opposed to this, the type of etch used for the oxide mask exhibits a much more pronounced influence. Both simulations and measurements demonstrate significantly smaller mask liftings for BHF etched masks compared to RIE etched masks. For all temperatures, the measured mask lifting is on average a factor of four higher for the RIE etched masks compared to the BHF etched masks. Although the analytical theory does not describe the BHF etched masks' geometry, this effect is to be expected, since the sloped mask edge created by the isotropic BHF etch impedes the oxidant diffusion from the side of the mask.

\section{Conclusions}

The topography of thermally oxidized, structured silicon dioxide was investigated through simulations, atomic force microscopy, and a proposed analytical model using conformal mapping. A $357 \mathrm{~nm}$ thick oxide was grown and structured by removing regions of the oxide in a masked etch with either reactive ion etching or hydrofluoric acid. Subsequent thermal oxidations were performed in both dry and wet ambient in the temperature range $950^{\circ} \mathrm{C}$ to $1100^{\circ} \mathrm{C}$ in order to grow a $205 \pm 12 \mathrm{~nm}$ thick oxide in the etched mask windows. The original oxide was seen to act as a diffusion mask during the second oxidation, resulting in lifting in the range $6 \mathrm{~nm}$ to $37 \mathrm{~nm}$ of the masking oxide near the edge of the mask. The mask lifting exhibited a slight oxidation parameter dependency, demonstrating increased lifting for increasing processing temperatures. The etch used to structure the oxide showed a much more pronounced effect on the mask lifting, with the reactive ion etched masks being lifted on average a factor of four more than oxides etched with hydrofluoric acid. Both simulations and the analytical model was able to predict the oxide topography qualitatively. The analytical model was used to derive a simple relationship between the mask lifting and the thicknesses of the oxide far from the mask edge. The simulations predicted the oxide topography quantitatively, with an average RMS deviation of $1.2 \mathrm{~nm}$ and a maximum deviation of $13 \mathrm{~nm} \mathrm{(39 \% )} \mathrm{from} \mathrm{the} \mathrm{mean} \mathrm{of} \mathrm{the} \mathrm{measured} \mathrm{values.}$

\section{Acknowledgments}

The authors thank Mathias Dahl Johnsen and Jeppe Nyskjold Lohse, Technical University of Denmark, for their help with sample preparation. This work was financially supported by the Danish National Advanced Technology Foundation (82-2012-4), and partly supported by the Danish National Research Foundation's Center for Individual Nanoparticle Functionality (DNRF54). 


\section{Appendix: Conformal Mapping of the Diffusion Problem}

The problem of finding the flux density in an oxide step similar to the structure investigated in this study can be solved through the use of conformal mapping. ${ }^{16}$ Let the infinite strip $0<\operatorname{Im} w<1$ in the complex plane represent a uniform oxide layer. The complex coordinates $w=\Psi+\mathrm{i} \mathcal{C}$ are used to represent the flux, $\Psi$, and concentration, $\mathcal{C}$, functions of the problem, respectively. The concentration at the top of the oxide at $\operatorname{Im} w=1$ is assumed to be $C_{0}$ during the whole oxidation, corresponding to a constant oxidant concentration in the furnace. The concentration at the silicon/oxide interface at $\operatorname{Im} w=0$ is assumed to be negligible and is set to zero. The real concentration is then given by $C=C_{0} \mathcal{C}$, while the real flux is $\psi=D C_{0} \Psi$.

The next step is to map the infinite strip on to a geometry similar to an oxide step. Such a geometry can be represented in the complex plane by $0<\operatorname{Im} z<1 \cap \operatorname{Re} z<0$, and $0<\operatorname{Im} z<1 / \alpha \cap \operatorname{Re} z>0$, where $z$ is a complex value and $\alpha=y_{\text {mask }} / y_{\text {window }}$, where $y_{\text {window }}$ is the thickness of the oxide grown in the mask windows and $y_{\text {mask }}$ is the thickness of the oxide mask as shown in Fig. 1. The conformal mapping from infinite strip to step geometry is performed in two steps: First, the infinite strip $0<\operatorname{Im} w<1$ is mapped on the upper half complex plane, $\xi$, which is accomplished through $\xi=\exp (\pi w)$ and $w=(\ln \xi) / \pi$. The mapping from the the half plane to the step geometry is subsequently accomplished through

$$
\begin{aligned}
z= & \frac{1}{\pi}\left[\ln \frac{\alpha^{2}-1}{\alpha^{2}+1-2 \sqrt{\xi-1} \sqrt{\xi-\alpha^{2}}-2 \xi}\right. \\
& \left.+\frac{i}{\alpha}\left(\frac{\pi}{2}+\operatorname{arctanh} \frac{\xi\left(\alpha^{2}+1\right)-2 \alpha^{2}}{2 \alpha \sqrt{\xi-1} \sqrt{\xi-\alpha^{2}}}\right)\right],
\end{aligned}
$$

with poles/zeroes at $\xi=0, \xi=1$, and $\xi=\alpha^{2}$ used in a Schwartz-Cristoffel transform. ${ }^{16}$ The complex coordinates $z=x+\mathrm{i} y$ represent the position in the oxide normalized to the thickness of the mask oxide, $y_{\text {mask }}$. Fig. 2 shows a plot of Eq. (A1) for $\alpha=5$, demonstrating iso-flux and iso-concentration contour lines. For convenience, the $x$-coordinate has been sign-inverted to orient the oxide mask to the right in accordance with the remaining figures in this paper.

The line $\operatorname{Im} w=1$ is mapped on $\operatorname{Im} z=0$, equivalent to the real axis, $x$, representing the oxide/silicon interface. The concentration at the silicon/oxide interface and at the oxide surface is thus interchanged in this mapping, but this does not affect the result, as it effectively corresponds to shifting the concentration's zero reference. At the silicon/oxide interface, $w=\Psi+\mathrm{i}$, corresponding to $\xi=-\exp (\pi \Psi)$. Inserting this in Eq. (A1) gives an expression for the position along the silicon/oxide interface $x$ as a function of the normalized flux $\Psi$ at the interface.

The parameter that determines the oxide growth is the normalized flux density at the silicon/oxide interface given by $\tilde{J}=d \Psi / d x$. By differentiation, it is seen that

$$
\frac{1}{\tilde{J}}=\frac{d x}{d \Psi}=-\sqrt{\frac{1+e^{\pi \Psi}}{\alpha^{2}+e^{\pi \Psi}}} \Rightarrow-e^{\pi \Psi}=\xi=\frac{\tilde{J}^{2}-\alpha^{2}}{\tilde{J}^{2}-1} .
$$

By inserting Eq. (A2) into Eq. (A1), the position at the interface normalized to $y_{\text {mask }}$ can be found as a function of normalized flux density $\tilde{J}$ :

$$
x=\frac{1}{\pi} \ln \left[\frac{\tilde{J}-1}{\tilde{J}+1}\left(\frac{\alpha+\tilde{J}}{\alpha-\tilde{J}}\right)^{1 / \alpha}\right] .
$$

The thickness of the grown oxide may now, in general, be calculated by finding the normalized flux density from Eq. (A3) and integrating Eq. (2) over time. However, at the edge of the step $x=0$, Eq. (A3) reduces to

$$
\frac{\tilde{J}_{0}+1}{\tilde{J}_{0}-1}=\left(\frac{\alpha+\tilde{J}_{0}}{\alpha-\tilde{J}_{0}}\right)^{1 / \alpha},
$$

where $\tilde{J}_{0}$ denotes the normalized flux density at the step edge. For $\alpha>1$, which is always true, Eq. (A4) yields an approximately constant relationship between the normalized flux density at the step edge $\tilde{J}_{0}$ and the thickness ratio $\alpha$ :

$$
\tilde{J}_{0} \simeq 0.83 \alpha \text {. }
$$

Hence, the real flux density at the mask edge $J_{0}$ becomes

$$
J_{0} \simeq 0.83 \frac{D C_{0} \alpha}{y_{\text {mask }}}=0.83 \frac{D C_{0}}{y_{\text {window }}}=0.83 J_{\text {window }},
$$

where the last equality comes from applying Fick's law $J=-D \nabla C$ to the onedimensional diffusion problem in the mask windows.

\section{References}

1. B. E. Deal and A. S. Grove, J. Appl. Physics, 36, 12 (1965).

2. H. Z. Massoud, J. D. Plummer, and E. A. Irene, J. Electrochem. Soc., 132, 7 (1985).

3. D. Chin, S.-Y. Oh, S.-M. Hu, R. W. Dutton, and J. L. Moll, IEEE Trans. Electron Devices, ED-30, 7 (1983).

4. T.-C. Wu, W. T. Stacy, and K. N. Ritz, J. Electrochem. Soc., 130, 7 (1983).

5. N. Guillemot, G. Pananakakis, and P. Chenevier, IEEE Trans. Electron Devices, ED-34, 5 (1987)

6. D.-B. Kao, J. P. McVittie, W. D. Nix, and K. C. Saraswat, IEEE Trans. Electron Devices, ED-34, 5 (1987).

7. D.-B. Kao, J. P. McVittie, W. D. Nix, and K. C. Saraswat, IEEE Trans. Electron Devices, ED-35, 1 (1988).

8. R. B. Marcus and T. T. Sheng, J. Electrochem. Soc., 129, 6 (1982).

9. K. Yamabe and K. Imai, IEEE Trans. Electron Devices, ED-34, 8 (1987).

10. A. F. Sarioglu, M. Kupnik, S. Vaithilingam, and B. T. Khuri-Yakub, J. Electrochem. Soc., 159, 2 (2012).

11. K. K. Park, H. Lee, M. Kupnik, and B. T. Khuri-Yakub, J. Microelectromech. Syst., 20, 1 (2011).

12. H.-S. Yoon, M.-C. Ho, N. Apte, P. Cristman, S. Vaithilingam, M. Kupnik, K. B. Pauly, and B. T. Khuri-Yakub, AIP Conf. Proc., 183 (2010).

13. T. L. Christiansen, O. Hansen, M. D. Johnsen, J. N. Lohse, J. A. Jensen, and E. V. Thomsen, Proc. IEEE Ultrason. Symp., 1737 (2013).

14. A. Plössl and G. Kräuter, Materials Science and Engineering, R25 (1999).

15. T. Martini, S. Hopfe, S. Mack, and U. Gösele, Sensors and Actuators A: Physical, 75 (1999).

16. W. Kaplan, Introduction to Analytic Functions, Addison-Wesley, Reading, MA (1966).

17. G. S. May and S. M. Sze, Fundamentals of Semiconductor Fabrication, p. 43, John Wiley \& Sons, New York (2004).

18. B. E. Deal, J. Electrochem. Soc., 125, 4 (1978).

19. R. R. Razouk, L. N. Lie, and B. E. Deal, J. Electrochem. Soc., 128, 10 (1981).

20. L. N. Lie, R. R. Razouk, and B. E. Deal, J. Electrochem. Soc., 129, 12 (1982).

21. ATHENA User's manual, Silvaco, Inc. p. 3-48 (2010).

22. E. P. EerNisse, Appl. Phys. Lett., 35, 1 (1979). 